\title{
Existence and uniqueness of mild solutions to the chemotaxis-fluid system modeling coral fertilization
}

Die Hu${ }^{1}$, Peng Chen ${ }^{1}$ and Deyi Ma ${ }^{1 *}$

${ }^{*}$ Correspondence:

mdysave@163.com

${ }^{1}$ College of Science, China Three

Gorges University, Yichang, P.R.

China

\begin{abstract}
In this paper, we consider the egg-sperm chemotaxis model of coral with the incompressible fluid equations in the whole space. The existence of global mild solutions in scaling invariant spaces is proved with sufficient small initial data. Here the main tool we use is the implicit function theorem. Furthermore, we obtain the asymptotic stability of solutions when the time goes to infinity. Since the initial data could be in the weak $L^{p}$-spaces, we finally get the existence of self-similar solutions when the initial data are small homogeneous functions.
\end{abstract}

Keywords: Chemotaxis-fluid system; Existence and uniqueness; Mild solutions; Self-similar solutions; Implicit function theorem

\section{Introduction and main results}

In this paper, we consider the following chemotaxis-fluid system modeling coral fertilization in $\mathbb{R}^{N}$ with $N \geq 3$ :

$$
\left\{\begin{array}{l}
\partial_{t} e+(\mathbf{u} \cdot \nabla) e-\Delta e=-\epsilon(s e), \\
\partial_{t} s+(\mathbf{u} \cdot \nabla) s-\Delta s=-\chi \nabla \cdot(s \nabla c)-\epsilon(s e), \\
\partial_{t} c+(\mathbf{u} \cdot \nabla) c-\Delta c=e, \\
\partial_{t} \mathbf{u}+k(\mathbf{u} \cdot \nabla) \mathbf{u}-\Delta \mathbf{u}+\nabla p=-(s+e) \nabla \phi, \quad \operatorname{div} \mathbf{u}=0,
\end{array}\right.
$$

where $e$ is the density of egg gametes, $s$ denotes the density of sperm gametes, $c$ represents the chemicals, $\mathbf{u}$ is the divergence free sea velocity of sea fluid, $\phi$ denotes the potential function, which is given by gravitational force and centrifugal force, the constants $\epsilon$ and $\chi$ are positive, and $k \in \mathbb{R}$. To complete system (1.1), the initial data are given by

$$
\left.e\right|_{t=0}=e_{0}(x),\left.\quad s\right|_{t=0}=s_{0}(x),\left.\quad c\right|_{t=0}=c_{0}(x), \quad \text { and }\left.\quad \mathbf{u}\right|_{t=0}=\mathbf{u}_{0}(x)
$$

(c) The Author(s) 2020. This article is licensed under a Creative Commons Attribution 4.0 International License, which permits use, sharing, adaptation, distribution and reproduction in any medium or format, as long as you give appropriate credit to the original author(s) and the source, provide a link to the Creative Commons licence, and indicate if changes were made. The images or other third party material in this article are included in the article's Creative Commons licence, unless indicated otherwise in a credit line to the material. If material is not included in the article's Creative Commons licence and your intended use is not permitted by statutory regulation or exceeds the permitted use, you will need to obtain permission directly from the copyright holder. To view a copy of this licence, visit http://creativecommons.org/licenses/by/4.0/. 
Here we see that system (1.1) is invariant by the transformation

$$
\begin{aligned}
& \bar{e}(x, t)=l^{2} e\left(l x, l^{2} t,\right), \quad \bar{s}(x, t)=l^{2} s\left(l x, l^{2} t,\right), \\
& \bar{c}(x, t)=c\left(l x, l^{2} t\right), \quad \overline{\mathbf{u}}(x, t)=l \mathbf{u}\left(l x, l^{2} t\right),
\end{aligned}
$$

up to a change of the pressure law $\bar{p}=l^{2} p$ for all $l>0$. And a function space is called critical space if the norm is invariant under transformation (1.3).

In the following, we will introduce the related works of this model. The most classical chemotaxis model describing the collective motion of cells or bacteria was first proposed by Patlak [1] and Keller and Segel [2,3]. In their papers, the partial differential equation of the random walk problem with orientation persistence and external bias was derived. In $[4,5]$, Kiselev and Ryzhik found that the fertilization rate could be close to completion as long as chemotaxis was strong enough. So they considered the case of the weakly coupled quadratic reaction term

$$
\partial_{t} n+(\mathbf{u} \cdot \nabla) n-\Delta n=\chi \nabla \cdot\left(n \nabla(\Delta)^{-1} n\right)-\varepsilon n^{q}, \quad \text { in }(x, t) \in \mathbb{R}^{d} \times(0, T) .
$$

Here, $n$ represents the density of egg (sperm) gametes, $\mathbf{u}$ is the specified spiral current rate, and $\chi>0$ denotes the chemotactic sensitivity constant, the term $\varepsilon n^{q}(q \geq 2)$ represents fertilization phenomenon. In $[6,7]$, under Neumann boundary condition, the authors provided a simpler proof of the nontrivial bounded classical solution of the decay profile for the following system:

$$
\left\{\begin{array}{l}
\partial_{t} n+(\mathbf{u} \cdot \nabla) n-\Delta n=-\chi \nabla \cdot(n \nabla c)-\varepsilon n^{2} \\
\partial_{t} c+(\mathbf{u} \cdot \nabla) c-\Delta c=-c+n
\end{array}\right.
$$

In [8], Espejo and Suzuki utilized the incompressible Navier-Stokes equation to describe the chemical $c$ and the velocity field $\mathbf{u}$ of fluid, which include the pressure $p$, and used $n \nabla \phi$ to simulate gravity. Those equations read as follows:

$$
\left\{\begin{array}{l}
\partial_{t} n+(\mathbf{u} \cdot \nabla) n-\Delta n=-\chi \nabla \cdot(n \nabla c)-\varepsilon n^{2}, \\
\partial_{t} c+(\mathbf{u} \cdot \nabla) c-\Delta c=-c+n, \\
\partial_{t} \mathbf{u}+k(\mathbf{u} \cdot \nabla) \mathbf{u}-\Delta \mathbf{u}+\nabla p=n \nabla \phi, \quad \operatorname{div} \mathbf{u}=0
\end{array}\right.
$$

Now, we consider a more general mathematical model (1.1) by making the egg density different from the sperm density in $\mathbb{R}^{N}$. For the chemotaxis-fluid system (1.1), many people have done a lot of research. For example, Chae, Kyungkeun, and Lee [9] studied the global well-posedness of coral fertilization models. Li, Pang, and Wang [10] explored the global boundedness and decay property. Zheng [11] showed the global weak solution of this system in a three-dimensional space. Htwe [12] proved the global classical-small data solutions. For more models based on the Navier-Stokes equations, one can refer to [1316] etc.

The aim of this paper is to prove the global existence of mild solutions to the chemotaxisfluid system (1.1) in $\mathbb{R}^{N}(N \geq 3)$ when the initial data are small in the scaling invariant spaces. Furthermore, based on our results concerning the existence and uniqueness of 
mild solutions (see Theorem 1.1), the global stability of those solutions is obtained under small initial perturbation. The main way we use is the implicit function theorem, which is inspired by Kozono, Miura, Sugiyama [17]. Let us mention that Tan, Wu, and Zhou [18] and Zhang, Deng, and Bie [19] applied the implicit function theorem to prove the existence and uniqueness of mild solutions to the magneto-hydro-dynamic equations and the nematic fluid crystals, respectively.

Before giving our results, we first introduce some usually used symbols and definitions of mild solutions. We denote $B C_{w}([0, \infty) ; X)$ as the set of bounded weakly-star continuous functions on $[0, \infty)$ with values in the Banach space $X$ and $L_{w}^{p}\left(\mathbb{R}^{N}\right)$ as the weak $L^{p}$-space.

Definition 1 Let $N \geq 3$, and assume that the initial data $\left\{e_{0}, s_{0}, c_{0}, \mathbf{u}_{0}\right\}$ satisfy $e_{0} \in L_{w}^{\frac{N}{2}}\left(\mathbb{R}^{N}\right)$, $s_{0} \in L_{w}^{\frac{N}{2}}\left(\mathbb{R}^{N}\right), \nabla c_{0} \in L_{w}^{N}\left(\mathbb{R}^{N}\right)$, and $\mathbf{u}_{0} \in L_{w}^{N}\left(\mathbb{R}^{N}\right)$. We call measurable function $\{e, s, c, \mathbf{u}\}$ on $\mathbb{R}^{N} \times(0, \infty)$ a mild solution of $(1.1)$ on $(0, \infty)$ if the identities

$$
\left\{\begin{aligned}
e(t)= & \exp (t \Delta) e_{0}-\int_{0}^{t} \exp \{(t-\tau) \Delta\}(\mathbf{u} \cdot \nabla) e(\tau) d \tau \\
& -\int_{0}^{t} \exp \{(t-\tau) \Delta\} \epsilon(s e)(\tau) d \tau, \\
s(t)= & \exp (t \Delta) s_{0}-\int_{0}^{t} \exp \{(t-\tau) \Delta\}(\mathbf{u} \cdot \nabla) s(\tau) d \tau \\
& -\int_{0}^{t} \exp \{(t-\tau) \Delta\} \epsilon(s e)(\tau) d \tau \\
& -\int_{0}^{t} \chi \nabla \cdot \exp \{(t-\tau) \Delta\}(s \nabla c)(\tau) d \tau, \\
c(t)= & \exp (t \Delta) c_{0}-\int_{0}^{t} \exp \{(t-\tau) \Delta\}(\mathbf{u} \cdot \nabla) c(\tau) d \tau+\int_{0}^{t} \exp \{(t-\tau) \Delta\} e(\tau) d \tau, \\
\mathbf{u}(t)= & \exp (t \Delta) u_{0}-\int_{0}^{t} \exp \{(t-\tau) \Delta\} P[k(\mathbf{u} \cdot \nabla) \mathbf{u}+(s+e) \nabla \phi](\tau) d \tau
\end{aligned}\right.
$$

hold for $t \in(0, \infty)$, where $\exp (t \Delta)$ denotes the heat semi-group defined by

$$
(\exp (t \Delta) g)(x) \equiv \int_{\mathbb{R}^{N}} G(x-y, t) g(y) d y
$$

with $G(x, t)=\frac{1}{(4 \pi t)^{\frac{N}{2}}} \exp \left(-\frac{|x|^{2}}{4 t}\right)$ and $P=\left\{P_{j k}\right\}_{j, k=1, \ldots, N}$ denotes the projection operator onto the solenoidal vector fields with the expression

$$
P_{j k}=\delta_{j k}+R_{j} R_{k} \quad\left(R_{j} \equiv \frac{\partial}{\partial x_{j}}(-\Delta)^{-\frac{1}{2}}: \text { Riesz operator }\right) \text { for } j, k=1,2, \ldots, N \text {. }
$$

Now our main results are as follows.

Theorem 1.1 (Existence) Let $N \geq 3$. Suppose that the indexes $p, q$, and $r$ satisfy

$$
q \geq 2, \quad \frac{N}{2}<q<N, \quad N<p<\frac{N q}{N-q}, \quad N<r<\frac{N q}{N-q},
$$

and there exists a constant $\delta=\delta(N, p, q, r)$ such that the initial data $\left\{e_{0}, s_{0}, c_{0}, \mathbf{u}_{0}\right\}$ satisfy the following condition:

$$
\begin{gathered}
\left\|e_{0}\right\|_{L_{w}^{\frac{N}{2}}}\left(\mathbb{R}^{N}\right)+\left\|s_{0}\right\|_{L_{w}^{\frac{N}{2}}}\left(\mathbb{R}^{N}\right)+\left\|\nabla c_{0}\right\|_{L_{w}^{N}}\left(\mathbb{R}^{N}\right) \\
+\left\|\mathbf{u}_{0}\right\|_{L_{w}^{N}}\left(\mathbb{R}^{N}\right)+\|\nabla \phi\|_{L_{w}^{N}}\left(\mathbb{R}^{N}\right)<\delta
\end{gathered}
$$


Then there exists a mild solution $\{e, s, c, \mathbf{u}\}$ of (1.1) which satisfies

$$
\begin{aligned}
& t^{\frac{N}{2}\left(\frac{2}{N}-\frac{1}{q}\right)} e \in B C_{w}\left([0, \infty) ; L^{q}\left(\mathbb{R}^{N}\right)\right), \\
& t^{\frac{N}{2}\left(\frac{2}{N}-\frac{1}{q}\right)} s \in B C_{w}\left([0, \infty) ; L^{q}\left(\mathbb{R}^{N}\right)\right), \\
& t^{\frac{N}{2}\left(\frac{1}{N}-\frac{1}{r}\right)} \nabla c \in B C_{w}\left([0, \infty) ; L^{r}\left(\mathbb{R}^{N}\right)\right), \\
& t^{\frac{N}{2}\left(\frac{1}{N}-\frac{1}{p}\right)} \mathbf{u} \in B C_{w}\left([0, \infty) ; L^{p}\left(\mathbb{R}^{N}\right)\right) .
\end{aligned}
$$

If the norms corresponding to spaces (1.11)-(1.14) are small enough, then the mild solution $\{e, s, c, \mathbf{u}\}$ to (1.1) is unique. Moreover, when $t \rightarrow \infty$, the mild solution $\{e, s, c, \mathbf{u}\}$ has the following asymptotic behavior:

$$
\begin{aligned}
& \left\|e(t)-\exp (t \Delta) e_{0}\right\|_{L^{q}}\left(\mathbb{R}^{N}\right)=O\left(t^{-\frac{N}{2}\left(\frac{2}{N}-\frac{1}{q}\right)}\right) \\
& \left\|s(t)-\exp (t \Delta) s_{0}\right\|_{L^{q}}\left(\mathbb{R}^{N}\right)=O\left(t^{-\frac{N}{2}\left(\frac{2}{N}-\frac{1}{q}\right)}\right) \\
& \left\|\nabla c(t)-\nabla \exp (t \Delta) c_{0}\right\|_{L^{r}}\left(\mathbb{R}^{N}\right)=O\left(t^{-\frac{N}{2}\left(\frac{1}{N}-\frac{1}{r}\right)}\right) \\
& \left\|\mathbf{u}(t)-\exp (t \Delta) \mathbf{u}_{0}\right\|_{L^{p}}\left(\mathbb{R}^{N}\right)=O\left(t^{-\frac{N}{2}\left(\frac{1}{N}-\frac{1}{p}\right)}\right)
\end{aligned}
$$

Theorem 1.2 (Stability) Let $N \geq 3$, and let the exponents $p, q$, and $r$ be the same as in Theorem 1.1, and $\delta=\delta(N, p, q, r)$ is the same constant as in (1.10). Suppose that the two initial data $\left\{e_{0}, s_{0}, c_{0}, \mathbf{u}_{0}\right\}$ and $\left\{\tilde{e}_{0}, \tilde{s}_{0}, \tilde{c}_{0}, \tilde{\mathbf{u}}_{0}\right\}$ and the two external forces $\phi$ and $\tilde{\phi}$ satisfy

$$
\begin{aligned}
& \left\|e_{0}\right\|_{L_{w}^{\frac{N}{2}}}\left(\mathbb{R}^{N}\right)+\left\|s_{0}\right\|_{L_{w}^{\frac{N}{2}}}\left(\mathbb{R}^{N}\right)+\left\|\nabla c_{0}\right\|_{L_{w}^{N}}\left(\mathbb{R}^{N}\right) \\
& +\left\|\mathbf{u}_{0}\right\|_{L_{w}^{N}}\left(\mathbb{R}^{N}\right)+\|\nabla \phi\|_{L_{w}^{N}}\left(\mathbb{R}^{N}\right)<\delta, \\
& \left\|\tilde{e}_{0}\right\|_{L_{w}^{\frac{N}{2}}}\left(\mathbb{R}^{N}\right)+\left\|\tilde{s}_{0}\right\|_{L_{w}^{\frac{N}{2}}}\left(\mathbb{R}^{N}\right)+\left\|\nabla \tilde{c}_{0}\right\|_{L_{w}^{N}}\left(\mathbb{R}^{N}\right) \\
& \quad\left\|\tilde{\mathbf{u}}_{0}\right\|_{L_{w}^{N}}\left(\mathbb{R}^{N}\right)+\|\nabla \tilde{\phi}\|_{L_{w}^{N}}\left(\mathbb{R}^{N}\right)<\delta .
\end{aligned}
$$

Suppose that $\{e, s, c, \mathbf{u}\}$ and $\{\tilde{e}, \tilde{s}, \tilde{c}, \tilde{\mathbf{u}}\}$ are mild solutions of $(1.1)$ on $[0, \infty)$ given by Theorem 1.1 with the initial data $\left\{e_{0}, s_{0}, c_{0}, \mathbf{u}_{0}\right\}$ and $\left\{\tilde{e}_{0}, \tilde{s}_{0}, \tilde{c}_{0}, \tilde{\mathbf{u}}_{0}\right\}$ in the class (1.11)-(1.14), respectively. Then, for any $\varepsilon>0$, there is a constant $\eta=\eta(N, p, q, r, \varepsilon)>0$ such that if

$$
\begin{gathered}
\left\|e_{0}-\tilde{e}_{0}\right\|_{L_{w}^{\frac{N}{2}}}+\left\|s_{0}-\tilde{s}_{0}\right\|_{L_{w}^{\frac{N}{2}}}+\left\|\nabla c_{0}-\nabla \tilde{c}_{0}\right\|_{L_{w}^{N}} \\
+\left\|\mathbf{u}_{0}-\tilde{\mathbf{u}}_{0}\right\|_{L_{w}^{N}}+\|\nabla \phi-\nabla \tilde{\phi}\|_{L_{w}^{N}}<\eta,
\end{gathered}
$$

then we have

$$
\begin{aligned}
& \sup _{0<t<\infty} t^{\frac{N}{2}\left(\frac{2}{N}-\frac{1}{q}\right)}\|e(t)-\tilde{e}(t)\|_{L^{q}}+\sup _{0<t<\infty} t^{\frac{N}{2}\left(\frac{2}{N}-\frac{1}{q}\right)}\|s(t)-\tilde{s}(t)\|_{L^{q}} \\
& \quad+\sup _{0<t<\infty} t^{\frac{N}{2}\left(\frac{1}{N}-\frac{1}{r}\right)}\|\nabla c(t)-\nabla \tilde{c}(t)\|_{L^{r}}+\sup _{0<t<\infty} t^{\frac{N}{2}\left(\frac{1}{N}-\frac{1}{p}\right)}\|\mathbf{u}(t)-\tilde{\mathbf{u}}(t)\|_{L^{p}}<\varepsilon .
\end{aligned}
$$

Corollary 1.1 (Self-similarity) Let $N \geq 3$, and suppose that the initial data $\left\{e_{0}, s_{0}, c_{0}, \mathbf{u}_{0}\right\}$ satisfy $e_{0} \in L_{w}^{\frac{N}{2}}, s_{0} \in L_{w}^{\frac{N}{2}}, \nabla c_{0} \in L_{w}^{N}$, and $\mathbf{u}_{0} \in L_{w}^{N}$. Assume that, for all $x \in \mathbb{R}^{N}$ and all $\lambda>0$, 
there are $e_{0}(\lambda x)=\lambda^{-2} e_{0}(x), s_{0}(\lambda x)=\lambda^{-2} s_{0}(x), c_{0}(\lambda x)=c_{0}(x), \mathbf{u}_{0}(\lambda x)=\lambda^{-1} \mathbf{u}_{0}(x)$, and $\phi(\lambda x)=$ $\phi(x)$. If the initial data $\left\{e_{0}, s_{0}, c_{0}, \mathbf{u}_{0}\right\}$ and $\nabla \phi$ satisfy condition (1.10), then the mild solution $\{e, s, c, \mathbf{u}\}$ given by Theorem 1.1 is a forward self-similar one, i.e., it holds that

$$
\begin{array}{lr}
e\left(\lambda x, \lambda^{2} t\right)=\lambda^{-2} e(x, t), & s\left(\lambda x, \lambda^{2} t\right)=\lambda^{-2} s(x, t), \\
c\left(\lambda x, \lambda^{2} t\right)=c(x, t), & \mathbf{u}\left(\lambda x, \lambda^{2} t\right)=\lambda^{-1} \mathbf{u}(x, t)
\end{array}
$$

for all $x \in \mathbb{R}^{N}, t>0$, and all $\lambda>0$.

\section{Key proposition}

Firstly, we introduce two function spaces $X$ and $Y$ defined as follows:

$$
X \equiv\left\{\left\{e_{0}, s_{0}, c_{0}, \mathbf{u}_{0}, \phi\right\} ; e_{0} \in L_{w}^{\frac{N}{2}}, s_{0} \in L_{w}^{\frac{N}{2}}, \nabla c_{0} \in L_{w}^{N}, \mathbf{u}_{0} \in L_{w}^{N}, \nabla \phi \in L_{w}^{N}\right\}
$$

with the norm

$$
\left\|\left(e_{0}, s_{0} c_{0}, \mathbf{u}_{0}, \phi\right)\right\|_{X} \equiv\left\|e_{0}\right\|_{L_{w}^{\frac{N}{2}}}+\left\|s_{0}\right\|_{L_{w}^{\frac{N}{2}}}+\left\|\nabla c_{0}\right\|_{L_{w}^{N}}+\left\|\mathbf{u}_{0}\right\|_{L_{w}^{N}}+\|\nabla \phi\|_{L_{w}^{N}}
$$

and

$$
\begin{aligned}
Y \equiv & \left\{\{e, s, c, \mathbf{u}\} ; t^{\frac{N}{2}\left(\frac{2}{N}-\frac{1}{q}\right)} e(\cdot) \in B C_{w}\left([0, \infty) ; L^{q}\right),\right. \\
& t^{\frac{N}{2}\left(\frac{2}{N}-\frac{1}{q}\right)} s(\cdot) \in B C_{w}\left([0, \infty) ; L^{q}\right), \\
& \left.t^{\frac{N}{2}\left(\frac{1}{N}-\frac{1}{r}\right)} \nabla c(\cdot) \in B C_{w}\left([0, \infty) ; L^{r}\right), t^{\frac{N}{2}\left(\frac{1}{N}-\frac{1}{p}\right)} \mathbf{u}(\cdot) \in B C_{w}\left([0, \infty) ; L^{p}\right)\right\}
\end{aligned}
$$

with the norm

$$
\begin{aligned}
& \|(e, s, c, \mathbf{u})\|_{Y} \\
& \equiv \sup _{0<t<\infty} t^{\frac{N}{2}\left(\frac{2}{N}-\frac{1}{q}\right)}\|e(t)\|_{L^{q}}+\sup _{0<t<\infty} t^{\frac{N}{2}\left(\frac{2}{N}-\frac{1}{q}\right)}\|s(t)\|_{L^{q}} \\
& \quad+\sup _{0<t<\infty} t^{\frac{N}{2}\left(\frac{1}{N}-\frac{1}{r}\right)}\|\nabla c(t)\|_{L^{r}}+\sup _{0<t<\infty} t^{\frac{N}{2}\left(\frac{1}{N}-\frac{1}{p}\right)}\|\mathbf{u}(t)\|_{L^{p}},
\end{aligned}
$$

respectively.

Here, $X$ and $Y$ are Banach spaces, and they have the norms $\|\cdot\|_{X}$ and $\|\cdot\|_{Y}$, respectively. For $\left\{e_{0}, s_{0} c_{0}, \mathbf{u}_{0}, \phi\right\} \in X$ and $\{e, s, c, \mathbf{u}\} \in Y$, we define the map

$$
F\left(e_{0}, s_{0}, c_{0}, \mathbf{u}_{0}, \phi, e, s, c, \mathbf{u}\right) \equiv\{E, S, C, U\}
$$


where

$$
\left\{\begin{aligned}
E(t)= & e(t)-\exp (t \Delta) e_{0}+\int_{0}^{t} \exp \{(t-\tau) \Delta\}(\mathbf{u} \cdot \nabla) e(\tau) d \tau \\
& +\int_{0}^{t} \exp \{(t-\tau) \Delta\} \epsilon(s e)(\tau) d \tau, \\
S(t)= & s(t)-\exp (t \Delta) s_{0}+\int_{0}^{t} \exp \{(t-\tau) \Delta\}(\mathbf{u} \cdot \nabla) s(\tau) d \tau \\
& +\int_{0}^{t} \exp \{(t-\tau) \Delta\} \epsilon(s e)(\tau) d \tau \\
& +\int_{0}^{t} \chi \nabla \cdot \exp \{(t-\tau) \Delta\}(s \nabla c)(\tau) d \tau, \\
C(t)= & c(t)-\exp (t \Delta) c_{0}+\int_{0}^{t} \exp \{(t-\tau) \Delta\}(\mathbf{u} \cdot \nabla) c(\tau) d \tau \\
& -\int_{0}^{t} \exp \{(t-\tau) \Delta\} e(\tau) d \tau, \\
U(t)= & \mathbf{u}(t)-\exp (t \Delta) \mathbf{u}_{0}+\int_{0}^{t} \exp \{(t-\tau) \Delta\} P[k(\mathbf{u} \cdot \nabla) \mathbf{u}+(s+e) \nabla \phi](\tau) d \tau, \\
0< & t<\infty .
\end{aligned}\right.
$$

Then we have the following key proposition.

Proposition 2.1 For $N \geq 3$, we assume that the exponents $p$, $q$, and $r$ satisfy the condition

$$
q \geq 2, \quad \frac{N}{2}<q<N, \quad N<p<\frac{N q}{N-q}, \quad N<r<\frac{N q}{N-q},
$$

then we deduce that:

(i) The map $F$ defined by (2.1) is a continuous map from $X \times Y$ into $Y$.

(ii) For each data $\left\{e_{0}, s_{0}, c_{0}, \mathbf{u}_{0}, \phi\right\} \in X$, the map $F\left(e_{0}, s_{0}, c_{0}, \mathbf{u}_{0}, \phi, \cdot, \cdot, \cdot, \cdot\right)$ is of class $C^{1}$ from $Y$ into itself.

Proof (i) First, we will verify that $t^{\frac{N}{2}\left(\frac{2}{N}-\frac{1}{q}\right)} E(t) \in B C_{w}\left([0, \infty) ; L^{q}\right)$. Owing to the $L^{q}-L_{w}^{N}$ estimate of the heat semi-group, it holds that

$$
\left\|\exp (t \Delta) e_{0}\right\|_{L^{q}} \leq C t^{-\frac{N}{2}\left(\frac{2}{N}-\frac{1}{q}\right)}\left\|e_{0}\right\|_{L_{w}^{\frac{N}{2}}}
$$

where $C=C(N, q)$, which means that $t^{\frac{N}{2}\left(\frac{2}{N}-\frac{1}{q}\right)} \exp (t \Delta) e_{0} \in B C_{w}\left([0, \infty) ; L^{q}\right)$.

According to (2.3), one has that $\frac{1}{2}-\frac{N}{2 p}>0, \frac{1}{N}<\frac{1}{p}+\frac{1}{q} \leq 1$. Then, for all $t>0$,

$$
\begin{aligned}
& \left\|\int_{0}^{t} \exp \{(t-\tau) \Delta\}(\mathbf{u} \cdot \nabla) e(\tau) d \tau\right\|_{L^{q}} \\
& \leq \int_{0}^{t}\|\nabla \cdot \exp \{(t-\tau) \Delta\}(\mathbf{u} e)(\tau)\|_{L^{q}} d \tau \\
& \leq C \int_{0}^{t}(t-\tau)^{-\frac{N}{2}\left(\frac{1}{q}+\frac{1}{p}-\frac{1}{q}\right)-\frac{1}{2}}\|\mathbf{u}(\tau)\|_{L^{p}}\|e(\tau)\|_{L^{q}} d \tau \\
& \leq C \sup _{0<\tau<\infty}\left(\tau^{\frac{N}{2}\left(\frac{2}{N}-\frac{1}{q}\right)}\|e(\tau)\|_{L^{q}}\right) \sup _{0<\tau<\infty}\left(\tau^{\frac{N}{2}\left(\frac{1}{N}-\frac{1}{p}\right)}\|\mathbf{u}(\tau)\|_{L^{p}}\right) \\
& \quad \times \int_{0}^{t}(t-\tau)^{\left(\frac{1}{2}-\frac{N}{2 p}\right)-1} \tau^{-\frac{1}{2}+\frac{N}{2}\left(\frac{1}{p}+\frac{1}{q}\right)-1} d \tau \\
& =C B\left(\frac{1}{2}-\frac{N}{2 p}, \frac{N}{2}\left(\frac{1}{p}+\frac{1}{q}\right)-\frac{1}{2}\right) \sup _{0<\tau<\infty}\left(\tau^{\frac{N}{2}\left(\frac{2}{N}-\frac{1}{q}\right)}\|e(\tau)\|_{L^{q}}\right)
\end{aligned}
$$




$$
\times \sup _{0<\tau<\infty}\left(\tau^{\frac{N}{2}\left(\frac{1}{N}-\frac{1}{p}\right)}\|\mathbf{u}(\tau)\|_{L^{p}}\right) t^{-\frac{N}{2}\left(\frac{2}{N}-\frac{1}{q}\right)}
$$

with $C=C(N, p, q)$, and here $B(s, t)$ denotes the beta function defined by

$$
B(s, t)=\int_{0}^{1} x^{s-1}(1-x)^{t-1} d x
$$

for positive constants $s$ and $t$.

Similarly, in view of $1-\frac{N}{2 q}>0,-1+\frac{N}{q}>0, \frac{1}{q}+\frac{1}{q} \leq 1$, we have

$$
\begin{aligned}
& \left\|\int_{0}^{t} \exp \{(t-\tau) \Delta\} \epsilon(s e)(\tau) d \tau\right\|_{L^{q}} \\
& \leq \int_{0}^{t}\|\exp \{(t-\tau) \Delta\} \epsilon(s e)(\tau)\|_{L^{q}} d \tau \\
& \leq C \int_{0}^{t}(t-\tau)^{-\frac{N}{2} \cdot \frac{1}{q}}\|s(\tau)\|_{L^{q}}\|e(\tau)\|_{L^{q}} d \tau \\
& \leq C \sup _{0<\tau<\infty}\left(\tau^{\frac{N}{2}\left(\frac{2}{N}-\frac{1}{q}\right)}\|s(\tau)\|_{L^{q}}\right) \\
& \times \sup _{0<\tau<\infty}\left(\tau^{\frac{N}{2}\left(\frac{2}{N}-\frac{1}{q}\right)}\|e(\tau)\|_{L^{q}}\right) \int_{0}^{t}(t-\tau)^{-\frac{N}{2} \cdot \frac{1}{q}} \tau^{\frac{N}{q}-2} d \tau \\
& =C B\left(1-\frac{N}{2 q},-1+\frac{N}{q}\right) \sup _{0<\tau<\infty}\left(\tau^{\frac{N}{2}\left(\frac{2}{N}-\frac{1}{q}\right)}\|s(\tau)\|_{L^{q}}\right) \\
& \times \sup _{0<\tau<\infty}\left(\tau^{\frac{N}{2}\left(\frac{2}{N}-\frac{1}{q}\right)}\|e(\tau)\|_{L^{q}}\right) t^{-\frac{N}{2}\left(\frac{2}{N}-\frac{1}{q}\right)}
\end{aligned}
$$

for all $t>0$ with $C=C(N, p, q)$.

Combining $(2.2)_{1}$ and $(2.4)-(2.6)$, we get $t^{\frac{N}{2}\left(\frac{2}{N}-\frac{1}{q}\right)} E(t) \in B C_{w}\left([0, \infty) ; L^{q}\right)$, and with the estimate

$$
\begin{aligned}
& \sup _{0<t<\infty} t^{\frac{N}{2}\left(\frac{2}{N}-\frac{1}{q}\right)}\|E(t)\|_{L^{q}} \\
& \leq C\left\|e_{0}\right\|{ }_{L_{w}^{\frac{N}{2}}}+C \sup _{0<\tau<\infty} \tau^{\frac{N}{2}\left(\frac{2}{N}-\frac{1}{q}\right)}\|e(\tau)\|_{L^{q}} \\
& \quad \times\left(1+\sup _{0<\tau<\infty} \tau^{\frac{N}{2}\left(\frac{1}{N}-\frac{1}{p}\right)}\|\mathbf{u}(\tau)\|_{L^{p}}+\sup _{0<\tau<\infty} \tau^{\frac{N}{2}\left(\frac{2}{N}-\frac{1}{q}\right)}\|s(\tau)\|_{L^{q}}\right),
\end{aligned}
$$

where $C=C(N, p, q)$.

Next, we are going to prove that $t^{\frac{N}{2}\left(\frac{2}{N}-\frac{1}{q}\right)} S(t) \in B C_{w}\left([0, \infty) ; L^{q}\right)$. In fact, it holds that

$$
\left\|\exp (t \Delta) s_{0}\right\|_{L^{q}} \leq C t^{-\frac{N}{2}\left(\frac{2}{N}-\frac{1}{q}\right)}\left\|s_{0}\right\|_{L_{W}^{\frac{N}{2}}}
$$

Since $\frac{1}{2}-\frac{N}{2 p}>0, \frac{N}{2}\left(\frac{1}{p}+\frac{1}{q}\right)-\frac{1}{2}>0, \frac{1}{p}+\frac{1}{q} \leq 1$ and $1-\frac{N}{2 q}>0,-1+\frac{N}{q}>0, \frac{1}{q}+\frac{1}{q} \leq 1$, we infer

$$
\begin{aligned}
& \left\|\int_{0}^{t} \exp \{(t-\tau) \Delta\}(\mathbf{u} \cdot \nabla) s(\tau) d \tau\right\|_{L^{q}} \\
& \quad \leq \int_{0}^{t}\|\nabla \cdot \exp \{(t-\tau) \Delta\}(\mathbf{u} s)(\tau)\|_{L^{q}} d \tau
\end{aligned}
$$




$$
\begin{aligned}
\leq & C \int_{0}^{t}(t-\tau)^{-\frac{N}{2}\left(\frac{1}{q}+\frac{1}{p}-\frac{1}{q}\right)-\frac{1}{2}}\|\mathbf{u}(\tau)\|_{L^{p}}\|s(\tau)\|_{L^{q}} d \tau \\
\leq & C \sup _{0<\tau<\infty}\left(\tau^{\frac{N}{2}\left(\frac{2}{N}-\frac{1}{q}\right)}\|s(\tau)\|_{L^{q}}\right) \sup _{0<\tau<\infty}\left(\tau^{\frac{N}{2}\left(\frac{1}{N}-\frac{1}{p}\right)}\|\mathbf{u}(\tau)\|_{L^{p}}\right) \\
& \times \int_{0}^{t}(t-\tau)^{\left(\frac{1}{2}-\frac{N}{2 p}\right)-1} \tau^{-\frac{1}{2}+\frac{N}{2}\left(\frac{1}{p}+\frac{1}{q}\right)-1} d \tau \\
= & C B\left(\frac{1}{2}-\frac{N}{2 p}, \frac{N}{2}\left(\frac{1}{p}+\frac{1}{q}\right)-\frac{1}{2}\right) \sup _{0<\tau<\infty}\left(\tau^{\frac{N}{2}\left(\frac{2}{N}-\frac{1}{q}\right)}\|s(\tau)\|_{L^{q}}\right) \\
& \times \sup _{0<\tau<\infty}\left(\tau^{\frac{N}{2}\left(\frac{1}{N}-\frac{1}{p}\right)}\|\mathbf{u}(\tau)\|_{L^{p}}\right) t^{-\frac{N}{2}\left(\frac{2}{N}-\frac{1}{q}\right)}
\end{aligned}
$$

and

$$
\begin{aligned}
& \left\|\int_{0}^{t} \exp \{(t-\tau) \Delta\} \epsilon(s e)(\tau) d \tau\right\|_{L^{q}} \\
& \leq \int_{0}^{t}\|\exp \{(t-\tau) \Delta\} \epsilon(s e)(\tau)\|_{L^{q}} d \tau \\
& \leq C \int_{0}^{t}(t-\tau)^{-\frac{N}{2} \cdot \frac{1}{q}}\|s(\tau)\|_{L^{q}}\|e(\tau)\|_{L^{q}} d \tau \\
& \leq C \sup _{0<\tau<\infty}\left(\tau^{\frac{N}{2}\left(\frac{2}{N}-\frac{1}{q}\right)}\|s(\tau)\|_{L^{q}}\right) \sup _{0<\tau<\infty}\left(\tau^{\frac{N}{2}\left(\frac{2}{N}-\frac{1}{q}\right)}\|e(\tau)\|_{L^{q}}\right) \\
& \quad \times \int_{0}^{t}(t-\tau)^{-\frac{N}{2} \cdot \frac{1}{q}} \tau^{\frac{N}{q}-2} d \tau \\
& =C B\left(1-\frac{N}{2 q},-1+\frac{N}{q}\right) \sup _{0<\tau<\infty}\left(\tau^{\frac{N}{2}\left(\frac{2}{N}-\frac{1}{q}\right)}\|s(\tau)\|_{L^{q}}\right) \\
& \quad \times \sup _{0<\tau<\infty}\left(\tau^{\frac{N}{2}\left(\frac{2}{N}-\frac{1}{q}\right)}\|e(\tau)\|_{L^{q}}\right) t^{-\frac{N}{2}\left(\frac{2}{N}-\frac{1}{q}\right)}
\end{aligned}
$$

for all $t>0$ with $C=C(N, p, q)$.

By a similar way, since $\frac{1}{2}-\frac{N}{2 r}>0, \frac{N}{2}\left(\frac{1}{r}+\frac{1}{q}\right)-\frac{1}{2}>0, \frac{1}{q}+\frac{1}{r} \leq 1$, one gets

$$
\begin{aligned}
& \left\|\int_{0}^{t} \chi \nabla \cdot \exp \{(t-\tau) \Delta\}(s \nabla c)(\tau) d \tau\right\|_{L^{q}} \\
& \leq \chi \int_{0}^{t}\|\nabla \cdot \exp \{(t-\tau) \Delta\}(s \nabla c)(\tau)\|_{L^{q}} d \tau \\
& \leq C \int_{0}^{t}(t-\tau)^{-\frac{N}{2}\left(\frac{1}{q}+\frac{1}{r}-\frac{1}{q}\right)-\frac{1}{2}}\|s \nabla c(\tau)\|_{L^{\frac{q r}{q+r}}} d \tau \\
& \leq C \int_{0}^{t}(t-\tau)^{-\frac{N}{2}\left(\frac{1}{q}+\frac{1}{r}-\frac{1}{q}\right)-\frac{1}{2}}\|s(\tau)\|_{L^{q}}\|\nabla c(\tau)\|_{L^{r}} d \tau \\
& \leq C \sup _{0<\tau<\infty}\left(\tau^{\frac{N}{2}\left(\frac{2}{N}-\frac{1}{q}\right)}\|s(\tau)\|_{\left.L^{q}\right)} \sup _{0<\tau<\infty}\left(\tau^{\frac{N}{2}\left(\frac{1}{N}-\frac{1}{r}\right)}\|\nabla c(\tau)\|_{L^{r}}\right)\right. \\
& \quad \times \int_{0}^{t}(t-\tau)^{\left(\frac{1}{2}-\frac{N}{2 r}\right)-1} \tau^{-\frac{1}{2}+\frac{N}{2}\left(\frac{1}{r}+\frac{1}{q}\right)-1} d \tau \\
& =C B\left(\frac{1}{2}-\frac{N}{2 r}, \frac{N}{2}\left(\frac{1}{r}+\frac{1}{q}\right)-\frac{1}{2}\right) \sup _{0<\tau<\infty}\left(\tau^{\frac{N}{2}\left(\frac{2}{N}-\frac{1}{q}\right)}\|\mathbf{s}(\tau)\|_{L^{q}}\right)
\end{aligned}
$$




$$
\times \sup _{0<\tau<\infty}\left(\tau^{\frac{N}{2}\left(\frac{1}{N}-\frac{1}{r}\right)}\|\nabla c(\tau)\|_{L^{r}}\right) t^{-\frac{N}{2}\left(\frac{2}{N}-\frac{1}{q}\right)}
$$

for all $t>0$ with $C=C(N, q, r)$.

Putting $(2.2)_{2}$ and (2.8)-(2.11) together, one has $t^{\frac{N}{2}\left(\frac{2}{N}-\frac{1}{q}\right)} S(t) \in B C_{w}\left([0, \infty) ; L^{q}\right)$, and with the estimate

$$
\begin{aligned}
& \sup _{0<t<\infty} t^{\frac{N}{2}\left(\frac{2}{N}-\frac{1}{q}\right)}\|S(t)\|_{L^{q}} \\
& \leq C\left\|s_{0}\right\|{ }_{L_{w}^{\frac{N}{2}}}+C \sup _{0<\tau<\infty} \tau^{\frac{N}{2}\left(\frac{2}{N}-\frac{1}{q}\right)}\|s(\tau)\|_{L^{q}} \\
& \quad \times\left(1+\sup _{0<\tau<\infty} \tau^{\frac{N}{2}\left(\frac{1}{N}-\frac{1}{p}\right)}\|\mathbf{u}(\tau)\|_{L^{p}}+\sup _{0<\tau<\infty} \tau^{\frac{N}{2}\left(\frac{2}{N}-\frac{1}{q}\right)}\|e(\tau)\|_{L^{q}}\right. \\
& \left.\quad+\sup _{0<\tau<\infty} \tau^{\frac{N}{2}\left(\frac{1}{N}-\frac{1}{r}\right)}\|\nabla c(\tau)\|_{L^{r}}\right)
\end{aligned}
$$

for all $t>0$, where $C=C(N, p, q, r)$.

In the following, we will demonstrate that $t^{\frac{N}{2}\left(\frac{1}{N}-\frac{1}{r}\right)} \nabla C(t) \in B C_{w}\left(0, \infty ; L^{r}\right)$. Indeed, it holds that

$$
\left\|\nabla \exp (t \Delta) c_{0}\right\|_{L^{r}} \leq C t^{-\frac{N}{2}\left(\frac{1}{N}-\frac{1}{r}\right)}\left\|\nabla c_{0}\right\|_{L_{w}^{N}}
$$

for all $t>0$. From (2.3), we have

$$
\begin{aligned}
& \left\|\nabla \int_{0}^{t} \exp \{(t-\tau) \Delta\}[(\mathbf{u} \cdot \nabla) c-e](\tau) d \tau\right\|_{L^{r}} \\
& \leq C \int_{0}^{t}(t-\tau)^{-\frac{N}{2}\left(\frac{1}{p}+\frac{1}{r}-\frac{1}{r}\right)-\frac{1}{2}}\|\mathbf{u}(\tau)\|_{L^{p}}\|\nabla c(\tau)\|_{L^{r}} d \tau \\
& +C \int_{0}^{t}(t-\tau)^{-\frac{N}{2}\left(\frac{1}{q}-\frac{1}{r}\right)-\frac{1}{2}}\|e(\tau)\|_{L^{q}} d \tau \\
& \leq C \sup _{0<\tau<\infty}\left(\tau^{\frac{N}{2}\left(\frac{1}{N}-\frac{1}{p}\right)}\|\mathbf{u}(\tau)\|_{L^{p}}\right) \sup _{0<\tau<\infty}\left(\tau^{\frac{N}{2}\left(\frac{1}{N}-\frac{1}{r}\right)}\|\nabla c(\tau)\|_{L^{r}}\right) \\
& \times \int_{0}^{t}(t-\tau)^{\left(\frac{1}{2}-\frac{N}{2 p}\right)-1} \tau^{\frac{N}{2}\left(\frac{1}{r}+\frac{1}{p}\right)-1} d \tau \\
& +C \sup _{0<\tau<\infty}\left(\tau^{\frac{N}{2}\left(\frac{2}{N}-\frac{1}{q}\right)}\|e(\tau)\|_{L^{q}}\right) \int_{0}^{t}(t-\tau)^{-\frac{N}{2}\left(\frac{1}{q}-\frac{1}{r}\right)-\frac{1}{2}} \tau^{\frac{N}{2 q}-1} d \tau \\
& =C B\left(\frac{1}{2}-\frac{N}{2 p}, \frac{N}{2}\left(\frac{1}{r}+\frac{1}{p}\right)\right) \\
& \times\left(\sup _{0<\tau<\infty} \tau^{\frac{N}{2}\left(\frac{1}{N}-\frac{1}{p}\right)}\|\mathbf{u}(\tau)\|_{L^{p}} \sup _{0<\tau<\infty} \tau^{\frac{N}{2}\left(\frac{1}{N}-\frac{1}{r}\right)}\|\nabla c(\tau)\|_{L^{r}}\right) t^{-\frac{N}{2}\left(\frac{1}{N}-\frac{1}{r}\right)} \\
& +C B\left(\frac{1}{2}-\frac{N}{2}\left(\frac{1}{q}-\frac{1}{r}\right), \frac{N}{2 q}\right)\left(\sup _{0<\tau<\infty} \tau^{\frac{N}{2}\left(\frac{2}{N}-\frac{1}{q}\right)}\|\mathbf{e}(\tau)\|_{L^{q}}\right) t^{-\frac{N}{2}\left(\frac{1}{N}-\frac{1}{r}\right)}
\end{aligned}
$$

for all $t>0$ with $C=C(N, p, q, r)$. Hence, it follows from $(2.2)_{3}$ and (2.13)-(2.14) that

$$
t^{\frac{N}{2}\left(\frac{1}{N}-\frac{1}{r}\right)} \nabla C(t) \in B C_{w}\left([0, \infty) ; L^{r}\right)
$$


with the estimate

$$
\begin{aligned}
\sup _{0<t<\infty} t^{\frac{N}{2}\left(\frac{1}{N}-\frac{1}{r}\right)}\|\nabla C(t)\|_{L^{r}} \\
\leq C\left\|\nabla c_{0}\right\|_{L_{w}^{N}}+C \sup _{0<\tau<\infty} \tau^{\frac{N}{2}\left(\frac{1}{N}-\frac{1}{r}\right)}\|\nabla c(\tau)\|_{L^{r}} \\
\quad+C \sup _{0<\tau<\infty} \tau^{\frac{N}{2}\left(\frac{1}{N}-\frac{1}{r}\right)}\|\nabla c(\tau)\|_{L^{r}} \sup _{0<\tau<\infty} \tau^{\frac{N}{2}\left(\frac{1}{N}-\frac{1}{p}\right)}\|\mathbf{u}(\tau)\|_{L^{p}} \\
\quad+C \sup _{0<\tau<\infty} \tau^{\frac{N}{2}\left(\frac{2}{N}-\frac{1}{q}\right)}\|e(\tau)\|_{L^{q}}
\end{aligned}
$$

for all $t>0$, where $C=C(N, p, q, r)$.

Finally, we deal with $U(t)$. Similar to (2.4), one has

$$
\left\|\exp (t \Delta) \mathbf{u}_{0}\right\|_{L^{p}} \leq C t^{-\frac{N}{2}\left(\frac{1}{N}-\frac{1}{p}\right)}\left\|\mathbf{u}_{0}\right\|_{L_{w}^{N}}
$$

for all $t>0$. By the fact that $\frac{1}{2}-\frac{N}{2 p}>0,1-\frac{N}{2}\left(\frac{1}{N}+\frac{1}{q}-\frac{1}{p}\right)>0$ and the boundedness of the projection operator $P$ in $L^{p}(1<p<\infty)$, we have

$$
\begin{aligned}
& \left\|\int_{0}^{t} \exp \{(t-\tau) \Delta\} P k(\mathbf{u} \cdot \nabla) \mathbf{u}(\tau) d \tau\right\|_{L^{p}} \\
& \quad \leq C \int_{0}^{t}\|\nabla \cdot \exp \{(t-\tau) \Delta\}(\mathbf{u} \otimes \mathbf{u})(\tau)\|_{L^{p}} d \tau \\
& \quad \leq C \int_{0}^{t}(t-\tau)^{-\frac{N}{2}\left(\frac{2}{p}-\frac{1}{p}\right)-\frac{1}{2}}\|(\mathbf{u} \otimes \mathbf{u})(\tau)\|_{L^{\frac{p}{2}}} d \tau \\
& \quad \leq C\left(\sup _{0<\tau<\infty} \tau^{\frac{N}{2}\left(\frac{1}{N}-\frac{1}{p}\right)}\|\mathbf{u}(\tau)\|_{L^{p}}\right)^{2} \int_{0}^{t}(t-\tau)^{\frac{1}{2}-\frac{N}{2 p}-1} \tau^{\frac{N}{p}-1} d \tau \\
& \quad=C B\left(\frac{1}{2}-\frac{N}{2 p}, \frac{N}{p}\right)\left(\sup _{0<\tau<\infty} \tau^{\frac{N}{2}\left(\frac{1}{N}-\frac{1}{p}\right)}\|\mathbf{u}(\tau)\|_{L^{p}}\right)^{2} t^{-\frac{N}{2}\left(\frac{1}{N}-\frac{1}{p}\right)}
\end{aligned}
$$

and

$$
\begin{aligned}
& \left\|\int_{0}^{t} \exp \{(t-\tau) \Delta\}(P(s+e) \nabla \phi)(\tau) d \tau\right\|_{L^{p}} \\
& \quad \leq C \int_{0}^{t}(t-\tau)^{-\frac{N}{2}\left(\frac{1}{N}+\frac{1}{q}-\frac{1}{p}\right)}\|s(\tau)\|_{L^{q}}\|\nabla \phi\|_{L_{w}^{N}} d \tau \\
& \quad+C \int_{0}^{t}(t-\tau)^{-\frac{N}{2}\left(\frac{1}{N}+\frac{1}{q}-\frac{1}{p}\right)}\|e(\tau)\|_{L^{q}}\|\nabla \phi\|_{L_{w}^{N}} d \tau \\
& \leq C\|\nabla \phi\|_{L_{w}^{N}} \sup _{0<\tau<\infty}\left(\tau^{\frac{N}{2}\left(\frac{2}{N}-\frac{1}{q}\right)}\|s(\tau)\|_{L^{q}}\right) \int_{0}^{t}(t-\tau)^{-\frac{N}{2}\left(\frac{1}{N}+\frac{1}{q}-\frac{1}{p}\right)} \tau^{\frac{N}{2 q}-1} d \tau \\
& \quad+C\|\nabla \phi\|_{L_{w}^{N}} \sup _{0<\tau<\infty}\left(\tau^{\frac{N}{2}\left(\frac{2}{N}-\frac{1}{q}\right)}\|e(\tau)\|_{L^{q}}\right) \int_{0}^{t}(t-\tau)^{-\frac{N}{2}\left(\frac{1}{N}+\frac{1}{q}-\frac{1}{p}\right)} \tau^{\frac{N}{2 q}-1} d \tau \\
& =C B\left(1-\frac{N}{2}\left(\frac{1}{N}+\frac{1}{q}-\frac{1}{p}\right), \frac{N}{2 q}\right)\left(\|\nabla \phi\|_{L_{w}^{N}} \sup _{0<\tau<\infty} \tau^{\frac{N}{2}\left(\frac{2}{N}-\frac{1}{q}\right)}\|s(\tau)\|_{L^{q}}\right) t^{-\frac{N}{2}\left(\frac{1}{N}-\frac{1}{p}\right)} \\
& \quad+C B\left(1-\frac{N}{2}\left(\frac{1}{N}+\frac{1}{q}-\frac{1}{p}\right), \frac{N}{2 q}\right)
\end{aligned}
$$




$$
\times\left(\|\nabla \phi\|_{L_{w}^{N}} \sup _{0<\tau<\infty} \tau^{\frac{N}{2}\left(\frac{2}{N}-\frac{1}{q}\right)}\|e(\tau)\|_{L^{q}}\right) t^{-\frac{N}{2}\left(\frac{1}{N}-\frac{1}{p}\right)}
$$

for all $t>0$ with $C=C(N, p, q)$.

Combining (2.2) 4 with (2.16)-(2.18), we have

$$
\sup _{0<\tau<\infty} t^{\frac{N}{2}\left(\frac{1}{N}-\frac{1}{p}\right)}\|U(\tau)\|_{L^{p}} \in B C_{w}\left(0, \infty ; L^{p}\right)
$$

with the estimate

$$
\begin{aligned}
\sup _{0<t<\infty} t^{\frac{N}{2}\left(\frac{1}{N}-\frac{1}{p}\right)}\|U(t)\|_{L^{p}} \\
\leq C\left\|\mathbf{u}_{0}\right\|_{L_{W}^{N}}+C \sup _{0<\tau<\infty} t^{\frac{N}{2}\left(\frac{1}{N}-\frac{1}{p}\right)}\|\mathbf{u}(\tau)\|_{L^{p}}\left(1+\sup _{0<\tau<\infty} \tau^{\frac{N}{2}\left(\frac{1}{N}-\frac{1}{p}\right)}\|\mathbf{u}(\tau)\|_{L^{p}}\right) \\
\quad+C\|\nabla \phi\|_{L_{w}^{N}} \sup _{0<\tau<\infty} \tau^{\frac{N}{2}\left(\frac{2}{N}-\frac{1}{q}\right)}\left(\|s(\tau)\|_{L^{q}}+\|e(\tau)\|_{L^{q}}\right)
\end{aligned}
$$

for all $t>0$, where $C=C(N, p, q)$.

Based on (2.7), (2.12), (2.15), and (2.19), we conclude that

$$
F\left(e_{0}, s_{0}, c_{0}, \mathbf{u}_{0}, \phi, e, s, c, \mathbf{u}\right) \equiv\{E, S, C, U\} \in Y
$$

with the estimate

$$
\begin{aligned}
& \| F\left(e_{0}, s_{0}, c_{0}, \mathbf{u}_{0}, \phi, e, s, c, \mathbf{u} \|_{Y}\right. \\
& \leq C\left\|\left\{e_{0}, s_{0}, c_{0}, \mathbf{u}_{0}, \phi\right\}\right\|_{X} \\
& \quad+C\|\{e, s, c, \mathbf{u}\}\|_{Y}\left(1+\|\{e, s, c, \mathbf{u}\}\|_{Y}+\|\nabla \phi\|_{L_{w}^{N}}\right),
\end{aligned}
$$

where $C=C(N, p, q, r)$. This means that $F$ is a continuous map from $X \times Y$ to $Y$.

(ii) We need to prove that $F$ is $C^{1}$. For each $\{e, s, c, \mathbf{u}\} \in Y$, we define a linear map $L_{\{e, s, c, \mathbf{u}\}}(\tilde{e}, \tilde{s}, \tilde{c}, \tilde{\mathbf{u}})=\{\tilde{E}, \tilde{S}, \tilde{C}, \tilde{U}\}$ on $Y$ by

$$
\left\{\begin{aligned}
\tilde{E}(t)= & \tilde{e}(t)+\int_{0}^{t} \exp \{(t-\tau) \Delta\}(\mathbf{u} \cdot \nabla) \tilde{e}(\tau) d \tau \\
& +\int_{0}^{t} \exp \{(t-\tau) \Delta\}(\tilde{\mathbf{u}} \cdot \nabla) e(\tau) d \tau \\
& +\int_{0}^{t} \exp \{(t-\tau) \Delta\} \epsilon(\tilde{s} e+s \tilde{e})(\tau) d \tau, \\
\tilde{S}(t)= & \tilde{s}(t)+\int_{0}^{t} \exp \{(t-\tau) \Delta\}[(\mathbf{u} \cdot \nabla) \tilde{s}+(\tilde{\mathbf{u}} \cdot \nabla) s](\tau) d \tau \\
& +\int_{0}^{t} \exp \{(t-\tau) \Delta\} \epsilon(\tilde{s} e+s \tilde{e})(\tau) d \tau \\
& +\int_{0}^{t} \chi \nabla \cdot \exp \{(t-\tau) \Delta\}(s \nabla \tilde{c}+\tilde{s} \nabla c)(\tau) d \tau, \\
\tilde{C}(t)= & \tilde{c}(t)+\int_{0}^{t} \exp \{(t-\tau) \Delta\}[(\mathbf{u} \cdot \nabla) \tilde{c}+(\tilde{\mathbf{u}} \cdot \nabla) c](\tau) d \tau \\
& -\int_{0}^{t} \exp \{(t-\tau) \Delta\} \tilde{e}(\tau) d \tau, \\
\tilde{U}(t)= & \tilde{\mathbf{u}}(t)+\int_{0}^{t} \exp \{(t-\tau) \Delta\} P[k(\mathbf{u} \cdot \nabla) \tilde{\mathbf{u}} \\
& +k(\tilde{\mathbf{u}} \cdot \nabla) \mathbf{u}+(\tilde{s}+\tilde{e}) \nabla \phi](\tau) d \tau .
\end{aligned}\right.
$$


We shall show that, for each fixed $\left\{e_{0}, s_{0}, c_{0}, \mathbf{u}_{0}, \phi\right\} \in X, L_{\{e, s, c, \mathbf{u}\}}$ is the Fréchet derivative of $F\left(e_{0}, s_{0}, c_{0}, \mathbf{u}_{0}, \phi, e, s, c, \mathbf{u}\right)$ at $\{e, s, c, \mathbf{u}\} \in Y$. We define $\{\mathfrak{E}, \mathfrak{S}, \mathfrak{C}, \mathfrak{U}\}$ by

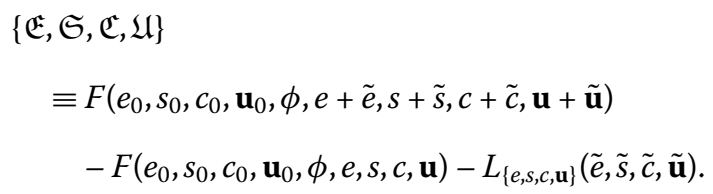

Then it holds that

$$
\begin{aligned}
\mathfrak{E}(t)= & e(t)+\tilde{e}(t)-\exp (t \Delta) e_{0}+\int_{0}^{t} \exp [(t-\tau) \Delta]([(\mathbf{u}+\tilde{\mathbf{u}}) \cdot \nabla](e+\tilde{e}))(\tau) d \tau \\
& +\int_{0}^{t} \exp [(t-\tau) \Delta](\epsilon(s+\tilde{s})(e+\tilde{e}))(\tau) d \tau \\
& -\left(e(t)-\exp (t \Delta) e_{0}+\int_{0}^{t} \exp [(t-\tau) \Delta](\mathbf{u} \cdot \nabla) e(\tau) d \tau\right. \\
& \left.+\int_{0}^{t} \exp [(t-\tau) \Delta] \epsilon(s e)(\tau) d \tau\right) \\
& -\left(\tilde{e}(t)+\int_{0}^{t} \exp \{(t-\tau) \Delta\}(\mathbf{u} \cdot \nabla) \tilde{e}(\tau) d \tau+\int_{0}^{t} \exp \{(t-\tau) \Delta\}(\tilde{\mathbf{u}} \cdot \nabla) e(\tau) d \tau\right. \\
& \left.+\int_{0}^{t} \exp \{(t-\tau) \Delta\} \epsilon(\tilde{s} e+s \tilde{e})(\tau) d \tau\right) \\
= & \int_{0}^{t} \exp \{(t-\tau) \Delta\}(\tilde{\mathbf{u}} \cdot \nabla) \tilde{e}(\tau) d \tau+\int_{0}^{t} \exp \{(t-\tau) \Delta\} \epsilon(\tilde{s} \tilde{e})(\tau) d \tau
\end{aligned}
$$

Hence, it follows from (2.5) and (2.6) that

$$
\begin{aligned}
\|\mathfrak{E}(t)\|_{L^{q}} \leq & C B\left(\frac{1}{2}-\frac{N}{2 p}, \frac{N}{2}\left(\frac{1}{p}+\frac{1}{q}\right)-\frac{1}{2}\right) \sup _{0<\tau<\infty}\left(\tau^{\frac{N}{2}\left(\frac{2}{N}-\frac{1}{q}\right)}\|\tilde{e}(\tau)\|_{L^{q}}\right) \\
& \times \sup _{0<\tau<\infty}\left(\tau^{\frac{N}{2}\left(\frac{1}{N}-\frac{1}{p}\right)}\|\tilde{\mathbf{u}}(\tau)\|_{L^{p}}\right) t^{-\frac{N}{2}\left(\frac{2}{N}-\frac{1}{q}\right)} \\
& +C B\left(1-\frac{N}{2 q},-1+\frac{N}{p}\right) \sup _{0<\tau<\infty}\left(\tau^{\frac{N}{2}\left(\frac{2}{N}-\frac{1}{q}\right)}\|\tilde{s}(\tau)\|_{L^{q}}\right) \\
& \times \sup _{0<\tau<\infty}\left(\tau^{\frac{N}{2}\left(\frac{2}{N}-\frac{1}{q}\right)}\|\tilde{e}(\tau)\|_{L^{q}}\right) t^{-\frac{N}{2}\left(\frac{2}{N}-\frac{1}{q}\right)}
\end{aligned}
$$

for all $t>0$.

In the same manner, we infer that

$$
\begin{aligned}
\mathfrak{S}(t)= & \int_{0}^{t} \exp \{(t-\tau) \Delta\}(\tilde{\mathbf{u}} \cdot \nabla) \tilde{s}(\tau) d \tau+\int_{0}^{t} \exp \{(t-\tau) \Delta\} \epsilon(\tilde{s} \tilde{e})(\tau) d \tau \\
& +\int_{0}^{t} \chi \nabla \cdot \exp \{(t-\tau) \Delta\}(\tilde{s} \nabla \tilde{c})(\tau) d \tau
\end{aligned}
$$

Putting (2.9), (2.10), and (2.11) together yields

$$
\|\mathfrak{S}(t)\|_{L^{q}} \leq C B\left(\frac{1}{2}-\frac{N}{2 p}, \frac{N}{2}\left(\frac{1}{p}+\frac{1}{q}\right)-\frac{1}{2}\right) \sup _{0<\tau<\infty}\left(\tau^{\frac{N}{2}\left(\frac{2}{N}-\frac{1}{q}\right)}\|\tilde{S}(\tau)\|_{L^{q}}\right)
$$




$$
\begin{aligned}
& \times \sup _{0<\tau<\infty}\left(\tau^{\frac{N}{2}\left(\frac{1}{N}-\frac{1}{p}\right)}\|\tilde{\mathbf{u}}(\tau)\|_{L^{p}}\right) t^{-\frac{N}{2}\left(\frac{2}{N}-\frac{1}{q}\right)} \\
& +C B\left(1-\frac{N}{2 q},-1+\frac{N}{p}\right) \sup _{0<\tau<\infty}\left(\tau^{\frac{N}{2}\left(\frac{2}{N}-\frac{1}{q}\right)}\|\tilde{s}(\tau)\|_{L^{q}}\right) \\
& \times \sup _{0<\tau<\infty}\left(\tau^{\frac{N}{2}\left(\frac{2}{N}-\frac{1}{q}\right)}\|\tilde{e}(\tau)\|_{L^{q}}\right) t^{-\frac{N}{2}\left(\frac{2}{N}-\frac{1}{q}\right)} \\
& +C B\left(\frac{1}{2}-\frac{N}{2 r}, \frac{N}{2}\left(\frac{1}{r}+\frac{1}{q}\right)-\frac{1}{2}\right) \sup _{0<\tau<\infty}\left(\tau^{\frac{N}{2}\left(\frac{2}{N}-\frac{1}{q}\right)}\|\tilde{s}(\tau)\|_{L^{q}}\right) \\
& \times \sup _{0<\tau<\infty}\left(\tau^{\frac{N}{2}\left(\frac{1}{N}-\frac{1}{r}\right)}\|\nabla \tilde{c}(\tau)\|_{L^{r}}\right) t^{-\frac{N}{2}\left(\frac{2}{N}-\frac{1}{q}\right)} .
\end{aligned}
$$

Similarly, for $\mathfrak{C}(t)$, it follows that

$$
\mathfrak{C}(t)=\int_{0}^{t} \exp \{(t-\tau) \Delta\}(\tilde{\mathbf{u}} \cdot \nabla) \tilde{c}(\tau) d \tau
$$

Then one gets from (2.14) that

$$
\begin{aligned}
&\|\nabla \mathfrak{C}(t)\|_{L^{r}} \\
& \leq C B\left(\frac{1}{2}-\frac{N}{2 p}, \frac{N}{2}\left(\frac{1}{r}+\frac{1}{p}\right)\right) \\
& \quad \times\left(\sup _{0<\tau<\infty} \tau^{\frac{N}{2}\left(\frac{1}{N}-\frac{1}{p}\right)}\|\tilde{\mathbf{u}}(\tau)\|_{L^{p}} \sup _{0<\tau<\infty} \tau^{\frac{N}{2}\left(\frac{1}{N}-\frac{1}{r}\right)}\|\nabla \tilde{c}(\tau)\|_{L^{r}}\right) t^{-\frac{N}{2}\left(\frac{1}{N}-\frac{1}{r}\right)} .
\end{aligned}
$$

Similar to (2.21), it holds that

$$
\mathfrak{U}(t)=\int_{0}^{t} \exp \{(t-\tau) \Delta\} P k(\tilde{\mathbf{u}} \cdot \nabla) \tilde{\mathbf{u}}(\tau) d \tau
$$

From (2.17), we get

$$
\|\mathfrak{U}(t)\|_{L^{p}} \leq C B\left(\frac{1}{2}-\frac{N}{2 p}, \frac{N}{p}\right)\left(\sup _{0<\tau<\infty} \tau^{\frac{N}{2}\left(\frac{1}{N}-\frac{1}{p}\right)}\|\tilde{\mathbf{u}}(\tau)\|_{L^{p}}\right)^{2} t^{-\frac{N}{2}\left(\frac{1}{N}-\frac{1}{p}\right)}
$$

for all $t>0$. Thus, by (2.22)-(2.25), we have

$$
\begin{aligned}
& \lim _{\| \tilde{e}, \tilde{s}, \tilde{c} \tilde{u}\} \|_{Y \rightarrow 0}} \frac{\|\{\mathfrak{E}, \mathfrak{S}, \mathfrak{C}, \mathfrak{U}\}\|_{Y}}{\|\{\tilde{e}, \tilde{s}, \tilde{c}, \tilde{\mathbf{u}}\}\|_{Y}} \\
& =\lim _{\|\left\{\tilde{e}, \tilde{s}, \tilde{\mathbf{u}}, \tilde{\mathbf{u}} \|_{Y} \rightarrow 0\right.}\left(\| F\left(e_{0}, s_{0}, c_{0}, \mathbf{u}_{0}, \phi, e+\tilde{e}, s+\tilde{s}, c+\tilde{c}, \mathbf{u}+\tilde{\mathbf{u}}\right)-F\left(e_{0}, s_{0}, c_{0}, \mathbf{u}_{0}, \phi, e, s, c, \mathbf{u}\right)\right. \\
& \left.-L_{\{e, s, c, \mathbf{u}\}}(\tilde{e}, \tilde{s}, \tilde{c}, \tilde{\mathbf{u}})\left\|_{Y} /\right\|\{\tilde{e}, \tilde{s}, \tilde{c}, \tilde{\mathbf{u}}\} \|_{Y}\right)=0
\end{aligned}
$$

for each $\left\{e_{0}, s_{0}, c_{0}, \mathbf{u}_{0}, \phi\right\} \in X$ and each $\{e, s, c, \mathbf{u}\} \in Y$. This implies that the Fréchet derivative of $F$ at point $\left\{e_{0}, s_{0}, c_{0}, \mathbf{u}_{0}, \phi, e, s, c, \mathbf{u}\right\} \in X \times Y$ in the direction to $\{e, s, c, \mathbf{u}\}$ is equal to $L_{\{e, s, c, \mathbf{u}\}}(\tilde{e}, \tilde{s}, \tilde{c}, \tilde{\mathbf{u}})$.

\section{Proof of the main results}

This section is devoted to proving Theorems 1.1-1.2 and Corollary 1.1. 
Proof of Theorem 1.1 Firstly, we shall show bijectivity of the Fréchet $L_{\{e, s, c, \mathbf{u}\}}$ at $\{e, s, c, \mathbf{u}\}=$ $\{0,0,0,0\}$. From (2.20), we have that $L_{\{0,0,0,0\}}\{\tilde{e}, \tilde{s}, \tilde{c}, \tilde{\mathbf{u}}\}=\left\{\tilde{E}_{0}, \tilde{S}_{0}, \tilde{C}_{0}, \tilde{\mathbf{U}}_{0}\right\}$, where

$$
\begin{aligned}
& \tilde{E}_{0}(t)=\tilde{e}(t), \quad \tilde{S}_{0}(t)=\tilde{s}(t), \quad \tilde{C}_{0}(t)=\tilde{c}(t)-\int_{0}^{t} \exp \{(t-\tau) \Delta\} \tilde{e}(\tau) d \tau, \\
& \tilde{\mathbf{U}}_{0}(t)=\tilde{\mathbf{u}}(t)+\int_{0}^{t} \exp \{(t-\tau) \Delta\} P[(\tilde{s}+\tilde{e}) \nabla \phi] d \tau
\end{aligned}
$$

for $\{\tilde{e}, \tilde{s}, \tilde{c}, \tilde{\mathbf{u}}\} \in Y$. Therefore, $\tilde{E}_{0}(t)=\tilde{S}_{0}(t)=\tilde{C}_{0}(t)=\tilde{\mathbf{U}}_{0}(t)=0$ implies that $\tilde{e}(t)=\tilde{s}(t)=\tilde{c}(t)=$ $\tilde{\mathbf{u}}(t)=0$, which means that $L_{\{0,0,0,0\}}$ is injective.

For each $\left\{\tilde{E}_{0}, \tilde{S}_{0}, \tilde{C}_{0}, \tilde{\mathbf{U}}_{0}\right\} \in Y$, we can take $\{\tilde{e}, \tilde{s}, \tilde{c}, \tilde{\mathbf{u}}\} \in Y$ as

$$
\begin{aligned}
& \tilde{e}(t)=\tilde{E}_{0}(t), \quad \tilde{s}(t)=\tilde{S}_{0}(t), \quad \tilde{c}(t)=\tilde{C}_{0}(t)+\int_{0}^{t} \exp \{(t-\tau) \Delta\} \tilde{E}_{0}(t) d \tau, \\
& \tilde{\mathbf{u}}(t)=\tilde{\mathbf{U}}_{0}(t)-\int_{0}^{t} \exp \{(t-\tau) \Delta\} P\left[\left(\tilde{S}_{0}(t)+\tilde{E}_{0}(t)\right) \nabla \phi\right] d \tau,
\end{aligned}
$$

then we obtain

$$
L_{\{0,0,0,0\}}\{\tilde{e}, \tilde{s}, \tilde{c}, \tilde{\mathbf{u}}\}=\left\{\tilde{E}_{0}, \tilde{S}_{0}, \tilde{C}_{0}, \tilde{\mathbf{U}}_{0}\right\}
$$

This means that $L_{\{0,0,0,0\}}$ is surjective from $Y$ to $Y$.

Now, using the Banach implicit function theorem, we could see that there exists a $C^{1}$ $\operatorname{map} h: X_{\delta} \rightarrow Y_{\delta}$,

$$
\begin{aligned}
& X_{\delta} \stackrel{\text { def }}{=}\left\{\left(e_{0}, s_{0}, c_{0}, \mathbf{u}_{0}, \phi\right) \in X ;\left\|\left(e_{0}, s_{0}, c_{0}, \mathbf{u}_{0}, \phi\right)\right\|_{X}<\delta\right\}, \\
& Y_{\delta} \stackrel{\text { def }}{=}\left\{(e, s, c, \mathbf{u}) \in Y ;\|(e, s, c, \mathbf{u})\|_{Y}<\delta\right\}
\end{aligned}
$$

for some $\delta(N, p, q, r)>0$ such that

$$
h(0,0,0,0)=\{0,0,0,0\}, \quad F\left(e_{0}, s_{0}, c_{0}, \mathbf{u}_{0}, \phi, h\left(e_{0}, s_{0}, c_{0}, \mathbf{u}_{0}, \phi\right)=\{0,0,0,0\}\right.
$$

for all $\left\{e_{0}, s_{0}, c_{0}, \mathbf{u}_{0}, \phi\right\} \in X_{\delta}$.

As a result, by condition (1.10), we may find that the function $h\left(e_{0}, s_{0}, c_{0}, \mathbf{u}_{0}, \phi\right)$ gives the unique solution of (1.7) satisfying properties (1.11)-(1.14).

The uniqueness of solutions $\{e, s, c, \mathbf{u}\}$ of (1.7) with the small norms corresponding to the class (1.11)-(1.14) is a consequence of the existence of the $C^{1}$-map $h$ from $X_{\delta}$ to $Y_{\delta}$. This shows that the asymptotic behaviors (1.15)-(1.18) follow from estimates (2.5) - (2.6), (2.9)-(2.11), (2.14), and (2.17)-(2.18), respectively.

Proof of Theorem 1.2 The estimate of stability (1.22) under condition (1.21) is a consequence of the continuity of the map $h: X_{\delta} \rightarrow Y_{\delta}$. The proof of Theorem 1.2 is complete.

Proof of Corollary 1.1 Assume that $\{e, s, c, \mathbf{u}\}$ is the solution of (1.7) by Theorem 1.1. Then one has

$$
e(x, t)=e_{1}(x, t)-E(e, s, c, \mathbf{u})(x, t), \quad s(x, t)=s_{1}(x, t)-S(e, s, c, \mathbf{u})(x, t),
$$




$$
c(x, t)=c_{1}(x, t)-C(e, s, c, \mathbf{u})(x, t), \quad \mathbf{u}(x, t)=\mathbf{u}_{1}(x, t)-U(e, s, c, \mathbf{u})(x, t),
$$

where

$$
\begin{array}{ll}
e_{1}(x, t)=\int_{\mathbb{R}^{N}} G(x-y, t) e_{0}(y) d y, & s_{1}(x, t)=\int_{\mathbb{R}^{N}} G(x-y, t) s_{0}(y) d y, \\
c_{1}(x, t)=\int_{\mathbb{R}^{N}} G(x-y, t) c_{0}(y) d y, & \mathbf{u}_{1}(x, t)=\int_{\mathbb{R}^{N}} G(x-y, t) \mathbf{u}_{0}(y) d y,
\end{array}
$$

and

$$
\begin{aligned}
E(e, s, c, \mathbf{u})(x, t)= & \int_{0}^{t} \int_{\mathbb{R}^{N}} G(x-y, t-\tau)(\mathbf{u} \cdot \nabla) e(y, \tau) d y d \tau \\
& +\int_{0}^{t} \int_{\mathbb{R}^{N}} G(x-y, t-\tau) \epsilon(s e)(y, \tau) d y d \tau, \\
S(e, s, c, \mathbf{u})(x, t)= & \int_{0}^{t} \int_{\mathbb{R}^{N}} G(x-y, t-\tau)(\mathbf{u} \cdot \nabla) s(y, \tau) d y d \tau \\
& +\int_{0}^{t} \int_{\mathbb{R}^{N}} G(x-y, t-\tau) \epsilon(s e)(y, \tau) d y d \tau \\
& +\int_{0}^{t} \int_{\mathbb{R}^{N}} \chi \nabla G(x-y, t-\tau)(s \nabla c)(\tau)(y, \tau) d y d \tau, \\
C(e, s, c, \mathbf{u})(x, t)= & \int_{0}^{t} \int_{\mathbb{R}^{N}} G(x-y, t-\tau)((\mathbf{u} \cdot \nabla) c(y, \tau)-e(y, \tau)) d y d \tau, \\
U_{i}(e, s, c, \mathbf{u})(x, t)= & \int_{0}^{t} \int_{\mathbb{R}^{N}} \sum_{j=1}^{N} E_{i j}(x-y, t-\tau) \\
& \times\left\{\sum_{k=1}^{N} k u_{k} \frac{\partial u_{j}}{\partial y_{k}}(y, \tau)+(s+e) \nabla \phi_{j}(y, \tau)\right\} d y d \tau
\end{aligned}
$$

for $i=1,2, \ldots, N$, with

$$
\begin{aligned}
& E_{i j}(y, \tau)=G(y, \tau) \delta_{i j}+\frac{\partial^{2}}{\partial_{i} \partial_{j}}[G(\cdot, \tau) * \Gamma](y), \quad i, j=1,2, \ldots, N, \\
& \Gamma(y)=\frac{1}{(N-2) \omega_{N}}|y|^{2-N} \quad \text { for } N \geq 3 .
\end{aligned}
$$

By the assumption on homogeneity of $e_{0}$, it holds that

$$
\begin{aligned}
e_{1}\left(\lambda x, \lambda^{2} t\right) & =\int_{\mathbb{R}^{N}} G\left(\lambda x-y, \lambda^{2} t\right) e_{0}(y) d y \\
& =\int_{\mathbb{R}^{N}} \frac{1}{\left(4 \pi \lambda^{2} t\right)^{N / 2}} \exp \left(-\frac{|\lambda x-y|^{2}}{4 \lambda^{2} t}\right) e_{0}(y) d y \\
& =\int_{\mathbb{R}^{N}} \frac{\lambda^{-N}}{(4 \pi t)^{N / 2}} \exp \left(-\frac{|x-z|^{2}}{4 t}\right) e_{0}(\lambda z) \lambda^{N} d z \\
& =\int_{\mathbb{R}^{N}} \frac{1}{(4 \pi t)^{N / 2}} \exp \left(-\frac{|x-z|^{2}}{4 t}\right) \lambda^{-2} e_{0}(z) d z=\lambda^{-2} e_{1}(x, t),
\end{aligned}
$$


where in the third equality, we have used the fact $z \stackrel{\text { def }}{=} \frac{y}{\lambda}$. Similarly, we have $s_{1}\left(\lambda x, \lambda^{2} t\right)=$ $\lambda^{-2} s_{1}(x, t), c_{1}\left(\lambda x, \lambda^{2} t\right)=c_{1}(x, t), \mathbf{u}_{1}\left(\lambda x, \lambda^{2} t\right)=\lambda^{-1} \mathbf{u}_{1}(x, t)$ for all $x \in \mathbb{R}^{N}, t>0$.

Since the solution $\{e, s, c, \mathbf{u}\}$ of system (1.7) in Theorem 1.1 is given by the mapping $h$ : $X_{\delta} \rightarrow Y_{\delta}$, in order to demonstrate Corollary 1.1, we will use the following proposition, and as its proof is quite standard, here we omit it.

Proposition 3.1 Let $N \geq 3$ and $\{e, s, c, \mathbf{u}\}$ satisfy (1.23). Then one has

$$
\begin{aligned}
& \lambda^{2} E(e, s, c, \mathbf{u})\left(\lambda x, \lambda^{2} t\right)=E(e, s, c, \mathbf{u})(x, t), \\
& \lambda^{2} S(e, s, c, \mathbf{u})\left(\lambda x, \lambda^{2} t\right)=S(e, s, c, \mathbf{u})(x, t), \\
& C(e, s, c, \mathbf{u})\left(\lambda x, \lambda^{2} t\right)=C(e, s, c, \mathbf{u})(x, t), \\
& \lambda U(e, s, c, \mathbf{u})\left(\lambda x, \lambda^{2} t\right)=U(e, s, c, \mathbf{u})(x, t)
\end{aligned}
$$

for all $x \in \mathbb{R}^{N}, t>0$ and all $\lambda>0$.

\section{Acknowledgements}

The authors would like to thank the anonymous referees for giving us helpful suggestions and comments which led to improvement of the presentation.

Funding

Research supported by the NNSF of China (Nos. 11871305, 11701325).

Availability of data and materials

Not applicable.

Competing interests

The authors declare that they have no competing interests.

Authors' contributions

$\mathrm{DH}, \mathrm{PC}$, and DM participated in theoretical research and drafted the manuscript. All authors read and approved the final manuscript.

\section{Publisher's Note}

Springer Nature remains neutral with regard to jurisdictional claims in published maps and institutional affiliations.

Received: 11 January 2020 Accepted: 26 March 2020 Published online: 06 April 2020

\section{References}

1. Patlak, C.S.: Random walk with persistence and external bias. Bull. Math. Biol. 15, 311-338 (1953)

2. Keller, E.F., Segel, L.A.: Initiation of slime mold aggregation viewed as an instability. J. Theor. Biol. 26, 399-415 (1970)

3. Keller, E.F., Segel, L.A.: Model for chemotaxis. J. Theor. Biol. 30, 225-234 (1971)

4. Kiselev, A., Ryzhik, L.: Biomixing by chemotaxis and enhancement of biological reactions. Commun. Partial Differ. Equ. 37, 298-318 (2012)

5. Kiselev, A., Ryzhik, L.: Biomixing by chemotaxis and efficiency of biological reactions: the critical reaction case. J. Math. Phys. 53, 53 (2012)

6. Htwe, M., Wang, Y.: Decay profile for the chemotactic model with advection and quadratic degradation in bounded domains. Appl. Math. Lett. 98, 36-40 (2019)

7. Cao, X., Winkler, M.: Sharp decay estimates in a bioconvection model with quadratic degradation in bounded domains. Proc. R. Soc. Edinb., Sect. A 148, 939-955 (2018)

8. Espejo, E., Suzuki, T.: Reaction terms avoiding aggregation in slow fluids. Nonlinear Anal., Real World Appl. 21, 110-126 (2015)

9. Chae, M., Kang, K., Lee, J.: Global well-posedness and long time behaviors of chemotaxis-fluid system modeling coral fertilization. arXiv:1904.06940

10. Li, J., Pang, P.Y.H., Wang, Y.: Global boundedness and decay property of a three-dimensional Keller-Segel-Stokes system modeling coral fertilization. Nonlinearity 32, 2815-2847 (2019)

11. Zheng, J.: Global weak solutions in a three-dimensional Keller-Segel-Navier-Stokes system modeling coral fertilization. arXiv:1905.08647

12. Htwe, M.: Global classical small-data solutions for a three-dimensional Keller-Segel-Navier-Stokes system modeling coral fertilization. arXiv:1907.01866 
13. Alghamdi, A.M., Gala, S., Ragusa, M.A.: On the blow-up criterion for incompressible Stokes-MHD equations. Results Math. 73, $110(2018)$

14. Gala, S., Ragusa, M.A.: A new regularity criterion for the 3D incompressible MHD equations via partial derivatives. J. Math. Anal. Appl. 481, 123497 (2020)

15. Saadati, R., Pourhadi, E., Samet, B.: On the PC-mild solutions of abstract fractional evolution equations with non-instantaneous impulses via the measure of noncompactness. Bound. Value Probl. 2019, 19 (2019)

16. Bie, Q., Wang, Q., Yao, Z.: Global well-posedness of the 3D incompressible MHD equations with variable density. Nonlinear Anal., Real World Appl. 47, 85-105 (2019)

17. Kozono, H., Miura, M., Sugiyama, Y.: Existence and uniqueness theorem on mild solutions to the Keller-Segel system coupled with the Navier-Stokes fluid. J. Funct. Anal. 270, 1663-1683 (2016)

18. Tan, Z., Wu, W., Zhou, J.: Existence and uniqueness of mild solutions to the magneto-hydro-dynamic equations. Appl. Math. Lett. 77, 27-34 (2018)

19. Zhang, Q., Deng, X., Bie, Q.: Existence and uniqueness of mild solutions to the incompressible nematic liquid crystal flow. Comput. Math. Appl. 77, 2489-2498 (2019)

Submit your manuscript to a SpringerOpen ${ }^{\circ}$ journal and benefit from:

- Convenient online submission

- Rigorous peer review

- Open access: articles freely available online

- High visibility within the field

- Retaining the copyright to your article

Submit your next manuscript at $\boldsymbol{\nabla}$ springeropen.com 